\title{
The Influence of Spine Health Exercise to the Fuction of Multifidus Muscle of Lumbosacral
}

\author{
Yan Xiao, Wang Yanqun, Li Kai \\ LinYi Normal University, LinYi, Shandong 276000, China
}

\begin{abstract}
To provide theoretical basis for the fitness value of spinal exercise by studying the influence of the spine exercise on multifidus muscle of lumbosacral. Selected 30 exercisers randomly which those age between 45-70 years on the basis of the normal physical activity remains the same, for at least four times a week spine exercises practitioners 15 people as experimental group, and the rest of the control group. Practice before 6 months after lumbar flexion buckling and straight average recorded in the process of electricity (ASEMG) and the average ratio (FER) electrical values on flexion and extension. The experimental group's ASEMG and FER of multifidus muscle of lumbosacral more significant differences before and after exercise. The spine exercise can increase the multifidus muscle function effectively.
\end{abstract}

KEYWORD: spine exercise; Multifidus Muscle; function

Currently, active motion therapy which cure mind lumbago mainly concentrate on active system to maintain the lumbar stability and control of lumbar posture ----- system improvement composed by muscle and tendon, in order to improve spine stability. The multifidus, which are closely linked with spine and fiber showed segmental division namely very short, is the main muscle groups to maintain spinal stability[1]. The stable fuction of Lumbosacral multifidus muscle to spine stabilization is largere than other segments, so the purpose of this research is according to the influence of the Spine Health Exercise on lumbosacral multifidus, providing a theoretical basis for the application of Spine Health Exercise in lumbago prevention, but also providing a theretical basis for the application of traditional exercise therapy in China.

\section{OBJECTS AND METHODS}

\subsection{OBJECTS}

Select exercisers in 15 as the experimental group in random whose age between 45 to 70 , years of practice for at least 1 year and at least 5 times a week to finish the whole movement of Spine Health Exercise in any park in Shijiazhuang, among them 7 males and 8 females. As Contrast group, choose exercisers in 15 at the age of 45 - 70 years old, who walk at least 30 minutes every day, among the 8 males and 7 females. No significant difference between the two groups of baseline data, so they can be compared in table 1 .

Table 1 basis data on objects

\begin{tabular}{|c|c|c|c|c|c|}
\hline groups & \multicolumn{2}{|c|}{ Gender } & age & height $(\mathrm{cm})$ & Weigh $(\mathrm{kg})$ \\
\hline & male & female & & & \\
\hline wuqixi & 7 & 8 & $63.625 \pm 3.068$ & $166.250 \pm 5.625$ & $66.750 \pm 7.869$ \\
\hline walking & 8 & 7 & $62.714 \pm 2.928$ & $165.429 \pm 7.892$ & $70.857 \pm 11.021$ \\
\hline \multicolumn{7}{|c|}{$\mathrm{t}$} & 0.586 & 0.235 & -0.839 \\
\hline & $\mathrm{p}$ & 0.568 & 0.818 & 0.416 \\
\hline
\end{tabular}




\subsection{Methods}

\subsubsection{Test equipment and analysis software:}

Biovision surface electromyography test system in German, analysis software is DASYLab10.0.

\subsubsection{Surface electrode plates and how it placed:}

Utility $\mathrm{Ag} / \mathrm{AgC} 1$ disposable electrode sheet surface made by Shanghai Zijunkang Medical Equipment Co., Ltd. According to the standard electrode position placed electrodes placed on the waist will L4 5 multifidus sites, and the center distance of $2 \mathrm{~cm}$ detection electrode, and reference electrode placed on the elbow olecranon, that is, the place where fat is least.

\subsubsection{Test position:}

Wth reference to the standard method of lumbar flexion, extension, the subjects stand straightly with eyes straight ahead and feet separated shoulder width, who slowly bending forward to maximize and then return to an upright position, stay $3 \mathrm{~S}$, repeat 3 times. Action process prompt feedback by computer voice, ensure the movement of batch consistency.

\subsubsection{Data acquisition and processing:}

Aplying Biovision surface electromyography test system nlarge the original EMG signal to 2500 times by the preamplifier. Gain lumbosacral flexion and extension in the the average surface EMG multifidus in trunk flexion process by applying the DASYLab10.0 analysis software. (average of surface electromyography, ASEMG). And according to a formula, calculate the average EMG flexion extension movements of flexion and extension ratio (flexion extension ratio, FER). FER= lumbar spine buckle in flexion process (The average surface EMG centrifugal) / the average surface EMG centripetal straight.

\subsection{Statistical analysis}

Use two independent samples t test and compare FER of multifidus muscle by SPSS13.0.

\section{RESULTS}

Values of ASEMG and FER of the experimental group and the control group in table 2 in the process of buckling of lumbar flexion and extension. The statistics show that two groups of subjects' ASEMG in buckling process was less than ASEMG in straightening process. This result is the same as Weijie's. The experimental group was lower than those in the control group in FER. Through t test, the flexion difference of the multifidus muscle on both sides all have prominent significance.

Table 2 ASEMG and FER in the process of the lumbar flexion and extension $(\mathrm{x} \pm \mathrm{s}, \mathrm{V})$

\begin{tabular}{|c|c|c|c|c|c|c|}
\hline \multirow[b]{2}{*}{ Group } & \multicolumn{3}{|c|}{ The left side of the multifidus muscle } & \multicolumn{3}{|c|}{ The right side of the multifidus muscle } \\
\hline & $\begin{array}{c}\text { Buckling of } \\
\text { ASEMG }\end{array}$ & $\begin{array}{c}\text { The extension of } \\
\text { ASEMG }\end{array}$ & FER & $\begin{array}{c}\text { Buckling of } \\
\text { ASEMG }\end{array}$ & $\begin{array}{c}\text { The extension of } \\
\text { ASEMG }\end{array}$ & FER \\
\hline $\begin{array}{c}\text { Spine Health Exercise } \\
\text { Group }\end{array}$ & $0.163 \pm 0.088$ & $0.287 \pm 0.126$ & $0.552 \pm 0.085$ & $0.177 \pm \quad 0.073$ & 0.2930 .109 & $0.616 \pm 0.138$ \\
\hline Vigorous walk group & $0.187 \pm 0.057$ & $0.276 \pm 0.100$ & $0.0686 \pm 0.088$ & $0.303 \pm 0.106$ & 0.3940 .151 & $0.781 \pm 0.083$ \\
\hline $\mathrm{t}$ & \multicolumn{3}{|c|}{-2.997} & \multicolumn{3}{|c|}{-2.751} \\
\hline
\end{tabular}

\section{DISCUSSION}

During the practice of Spine Health Exercise, the movement of the spine drives the whole body movement, while lumbar activity is the major part in the movement of the spine, it includes lumbar flexion, extension, lateral flexion, rotation and ring turn to make back muscles stretch in different directions, meanwhile flexion and rotation can make the multifidus muscle contract, extension can make the multifidus muscle relax; This provides the prerequisites for researching the use of Spine Health Exercise in the prevention of lumbago.

The study put emphasis on the research that uses Spine Health Exercise in Lumbosacral multifidus muscle[2]. The reason has two sides. On the one hand, the lumbosacral multifidus muscle is the most developed muscle in our body, it has the largest function in the lumbar stability; Since the sacrum back above the inwards oblique, superficial muscle bundle stops above the 3-4 vertebrae spinous process, the middle layer of the muscle bundle stops above the 2-3 vertebrae spinous process, the deepest bundles connected on the upper part of the adjacent vertebrae. On the other hand, currently the active movement treatment method in curing lumbago mainly focuses on the research which studies the local stability of multifidus and abdominal muscle in deep trunk; the result shows that multifidus and abdominal muscle has the common contraction system, while multifidus belongs to lumbar back muscles, so the treatment of lumbago mainly focuses on researching multifidus, and there is enough evidence to prove its important role in the stability of 
the spine. For example, Liu Bangzhong and some other persons found that compared with the erector spinae and lumbar iliocostal muscles, the multifidus muscle reacts more quickly when the spine has sudden imbalance[3]; Recently, many scholars switch emphasis to study deep fibres of lumbar multifidus (DM) and summarize five clinical evidences. These evidences are DM's role in the lumbar activity and its importance in the pathogenesis of lumbago pain[4].

The surface electromyogram (EMG) is electrophysiological signals which record muscle activity from the body surface, it can reflect muscle activity state, thus evaluate the function of muscle. The recent studies show, the function of using EMG to evaluate back muscle gradually improves, and has a certain reliability, specificity and sensitivity; Among them FER is a positive index in the evaluation of the function of low back muscle. FER is an adaptation index reflecting the waist motion, its abnormal shows neuromuscular coordination between the trunk and hip may also be abnormal. The multifidus muscle as the antagonist muscle in the buckling process of the process of spinal flexion, as the active muscle in the straightening process, while active movement works more than antagonistic sports and produces higher surface EMG. So the buckling of ASEMG was less than straight ASEMG. The increase of FER represents the reduce of the muscles' active power, or the increase of antagonistic power, all this show that the muscle itself has function problem, that means at the completion of the same tasks other muscles have compensatory effects; At present, many scholars study the relationship between paraspinal muscle FER and lumbago[5]. So the research uses this index as the index of the multifidus muscle function change.

Compared with the Spine Health Exercisers and vigorous walk persons, the reason of Spine Health Exercise's obvious function in lumbosacral multifidus muscle is that in the process of practicing Spine Health Exercise, the constant change in the center of gravity lead to unbalanced state of the body. When completing the action, the trunk need to stay in the state of imbalance and keep a certain time, during the middle process, waist deep local muscles, that is, the multifidus muscle need to overcome the external load acting in order to maintain the balance of the body, when the action finishes, not only the multifidus muscle needs to overcome the external work, but also calls for additional work to maintain the body posture stability. Meanwhile the action of Spine Health Exercise is coherent and changeable, this effectively stimulates the central nervous system's control over the multifidus muscle, thus trains its function more effectively. This is consistent with training core muscle theory at present. In the movement of Spine
Health Exercise, it contains the dorsiflexion action to relax the multifidus muscle effectively, this can make the multifidus muscle combine between contraction and relaxation, so as not to let the multifidus muscle be fatigued. The multifidus muscle, is the muscle which most close to the midline of lumbar paraspinal muscle and closely connected with the vertebral muscle. Its main role is to against spinal rotation and sliding rather than create a spinal action, Research has shown that stimulation of porcine intervertebral disc and facet joint capsule can produce the multifidus muscle contraction. During the practice process of Spine Health Exercise, the every direction of the spine has been effectively drafted, such as tiger lift, tiger fiutter, deer run, monkey pick and so on. While the draft action due to vertebral activities can effectively stimulate the disc, articular capsule, etc; In addition, the direction of rotation of the sliding on drafting spine caused by rotation and vertebral body, thus lead to the contraction of the multifidus muscle; Action after reduction can make it relax. This is consistent with the principles of strength training and muscle stretching and can make the multifidus muscle have effective training. However, the center of gravity changes is relatively stable in the process of walking, so The multifidus muscle is at the same condition for a long time, that is, the state of contraction. It is possible to aggravate the fatigue state of the multifidus muscle, which causes its function decease because rarely use the dorsiflexion action in daily life and work.

Now, Lumbar vertebra MRI shows: There significant correlation between the multifidus muscle atrophy and lumbago. Many scholars have tried to use the multifidus muscle imaging changes to evaluate and guide the treatment of lumbago. Histological study found that there existed changes in low back pain patients' the multifidus muscle size and muscle fiber type. Other researches show that the multifidus muscle atrophy and low back pain have mutual cause-and-effect relationship-----the multifidus muscle atrophy can aggravate the pain or increase the recurrence rate of lumbago and low back pain increases muscle atrophy. Spine Health Exercise can improve the function of multifidus muscle; thereby break up this vicious spiral, and then relief lumbago.

Degenerative lumbar can cause spinal instability that increases its activity, the paravertebral muscles become slack for lack of scientific training, which are not strong enough to against instability caused by degeneration, thus lead to lumbago[6]. Currently, lumbar disc degeneration is irresistible, but we can against this instability through training the function of paravertebral muscle[7]. Improving the paraspinal muscle function especially for multifidus muscle can increase the stability of the spine; recover the normal physiological curvature of vertebral column; which 
make spinal mechanics and intervertebral joint relatively stable, therefore lighten pain caused by soft tissue injury that result from joint laxity, shift, stimulate of nerve endings, and then release lumbago until vanish.

\section{ACKNOWLEDGEMENTS}

This work was supported by the natural science foundation of Shandong Province. Item Number is ZR2010HL001.

\section{REFERENCES}

[1] Panjabi MM. The stabilizing system of the spine. PartI. Function, dysfunction, adaptation, and enhancement. J Spinal Disord, 1992, (4):383-389.
[2] McGill SM. low back stability: from formal description to issues for performance and rehabilitation. Exercise and Sport Science Reviews, 2001, 9(1):26-31.

[3] Lee SW,Wong KW, Chan MK, et al. Development and validation of a new technique for assessing lumbar spine motion. Spine, 2002, 27(8): E215-E220.

[4] Ritvanen T, Zaproudina N, Nissen M, et al. Dynamic surface electromyographicresponses in chronic low back pain treated by traditional bone setting and conventional physical therapy. J Manipulative Physiol Ther, 2007, 30(1):31-37.

[5] MacDonald DA, Moseley GL, Hodges PW. The lumbar multifidus: Does the evidence support clinical beliefs? Manual Therapy, 2006, (11):254-263.

[6] Dankaerts W, O'Sullivan PB, Burnett AF, et al. Reliability of EMG measurements for trunk muscles during maximal and sub maximal voluntary isometric contractions in healthy controls and CLBP patients. J Electromyogr Kinesiol, 2004, 14(3):333-342.

[7] Mehling WE, Hamel KA, Acree M,et al. Randomized, controlled trial of breath therapy for patient with chronic low back pain. Altemative Therapies in Health and Medicine, 2005, 11: 44-52. 